\section{A) Check for updates}

Cite this: Food Funct., 2019, 10, 6193

\title{
Glycation affects differently the main soybean Bowman-Birk isoinhibitors, IBB1 and IBBD2, altering their antiproliferative properties against HT29 colon cancer cells
}

\author{
Raquel Olías, (D) *a Carmen Becerra-Rodríguez, (D) a Jorge R. Soliz-Rueda, (D) a \\ F. Javier Moreno, (D) ${ }^{b}$ Cristina Delgado-Andrade (iD ${ }^{c}$ and Alfonso Clemente (iD ${ }^{a}$
}

\begin{abstract}
Naturally-occurring serine protease inhibitors of the Bowman-Birk family, particularly abundant in legume seeds, exert their potential chemopreventive and/or therapeutic properties via protease inhibition. Processing of legume seeds, including soybeans, has been proposed as a major cause for their loss of bioactivity due to glycation. In order to assess how glycation affected the protease inhibitory activities of major soybean Bowman-Birk isoinhibitors (BBI) and their antiproliferative properties, IBB1 and IBBD2 were purified and subjected to glycation under controlled conditions using glucose at high temperature. Both soybean isoinhibitors showed remarkable heat stability. In the presence of glucose, IBBD2 lost most of its trypsin inhibitory activity while IBB1 maintains similar trypsin and chymotrypsin inhibitory activities as in the absence of sugar. Glycation patterns of both BBI proteins were assessed by MALDI-TOF spectrometry. Our results show that the glycation process affects IBBD2, losing partially its antiproliferative activity against HT29 colon cancer cells, while glycated-IBB1 was unaffected.
\end{abstract}

Received 1st July 2019,

Accepted 30th August 2019

DOI: $10.1039 /$ c9fo01421g

rsc.li/food-function to the number and distribution of intramolecular disulphide bonds is mostly responsible for the high stability of these proteins towards extreme conditions and helps to maintain the structural and functional features of their binding loops. ${ }^{3-5}$

Colorectal cancer is the third leading cause of cancerrelated death in the United States and emerging evidence supports that increased consumption of legumes can reduce risk. ${ }^{6}$ Among other bioactive compounds, several in vitro and in vivo studies have demonstrated that BBI proteins from soybean and related legume species may exert a protective and/or suppressive effect in colorectal cancer development and associated inflammatory disorders. ${ }^{7-9}$ The emerging evidence suggests that BBI exert their anti-proliferative properties via protease inhibition. ${ }^{10}$ In particular, the anti-carcinogenic properties of soybean BBI have been linked to the chymotrypsin inhibitory domain, leading to the hypothesis that chymotrypsin-like proteases are potential targets of BBI. In contrast we have demonstrated that IBBD2, which inhibits trypsin-like proteases only, exerts anti-proliferative properties against colon cancer cells. ${ }^{1}$

Many different foods, legumes as well, are usually subjected to different industrial and/or domestic processing involving thermal treatment. During those processes, amino-carbonyl reaction spontaneously takes place in foods containing both amino and carbonyl groups; indeed, food proteins react with reducing sugars to form glycated proteins, resulting in structural changes that may dramatically affect the biological 
activity of proteins. ${ }^{11}$ The functional properties of BBI seem to be affected by glycation. Thus, the decrease in the trypsin inhibitory activity of the products obtained by the amino-carbonyl reaction between several trypsin inhibitors and glucose, at $50{ }^{\circ} \mathrm{C}$ and $65 \%$ relative humidity, was established some time ago. Moreover, the decline in the trypsin inhibitory activity of lysine-type inhibitors, BBI among them, was tightly correlated to the drop in free amino and guanidine groups of the proteins. ${ }^{12}$ More recently, Amigo-Benavent et al. (2013) pointed to glycation during sterilization of orange juice enriched with soybean BBI as the responsible for the changes detected in the bioactivity of this protease inhibitor. ${ }^{13}$ Arques et al. (2016) suggested that BBI glycation under heat treatment might be responsible for the decrease of protease inhibitory activity in soymilks. ${ }^{14}$ These authors also established the role of furosine, a known marker of Maillard reaction and glycation, as a potential indicator to monitor both thermal treatment and effects on protease inhibitory activities in soy milks. Other researchers, studying the loss of inhibitory activity of BBI during the manufacturing of soymilk, proposed that this was a very complex process and involved protein aggregation of BBI with other proteins present in the food matrix as responsible for the loss of activity. ${ }^{15,16}$ Apart from the above mentioned, the scientific literature describing the effect of glycation on the bioactivity of protease inhibitors is scarce. No information has been reported regarding the mechanisms that lead to the loss of the BBI functionality, the preferential sites for glycation or if it affects homogenously to the major BBI isoforms, each of them with different selectivity in its protease inhibitory activities. Since many aspects remain to be elucidated, we have focused on boiling-like operations as a model to evaluate the glycation process of BBI isoforms, as this culinary procedure is widely applied by food industry and home cooking to different foods, BBI sources among them. Therefore, the aim of this work was to determine the occurrence of glycation and the glycation sites of the two major BBI isoforms from soybean, IBB1 and IBBD2 under controlled conditions (similar to boiling) using a kinetical design to evaluate the key moments for glycation during the time course. The effects of glycation on the protease inhibitory activities of both isoforms as well as on their antiproliferative effect against HT29 human colorectal adenocarcinoma cells were assessed.

\section{Material and methods}

\subsection{Materials}

BBI (T9777) from soybean, trypsin (type III, T1436) and $\alpha$-chymotrypsin (type VII, C3142) from bovine pancreas, $N$ - $\alpha$-benzoyl-DL-arginine- $p$-nitroanilide (BAPNA, B4875) and $N$-benzoyl-L-tyrosine ethyl ester (BTEE,) were obtained from Sigma-Aldrich (Alcobendas, Spain). The human colorectal adenocarcinoma HT29 cell line was supplied by the Cell Bank of the Scientific Instrumentation Centre at the University of Granada (CIC-UGR, Granada, Spain). Culture flasks and flat bottom 96-well microtiter plates were purchased from Corning
Costar (Cambridge, MA, USA) and Nunc (Wiesbaden, Germany), respectively. All other chemicals were of analytical grade.

\subsection{Isolation of major soybean protease isoinhibitors}

The major BBI isoinhibitors, IBB1 and IBBD2, were purified from commercially available soybean BBI using a MonoS 5/50 GL cation exchange column (GE Healthcare, Uppsala, Sweden), connected to an AKTA FPLC system (GE Healthcare). Soybean BBI was prepared by dissolving $4 \mathrm{mg}$ of BBI in $6 \mathrm{~mL}$ of $50 \mathrm{mM}$ sodium acetate buffer, $\mathrm{pH}$ 4.4. The elution was performed using a linear gradient of $0-0.22 \mathrm{M} \mathrm{NaCl}$ in $50 \mathrm{mM}$ sodium acetate buffer, $\mathrm{pH} 4.4$, at a flow rate of $1 \mathrm{~mL} \mathrm{~min}^{-1}$. The elution pattern of the mixture of protease inhibitors was monitored at $280 \mathrm{~nm}$ and $0.5 \mathrm{~mL}$ fractions were collected. Trypsin inhibitory activity (TIA) measurements of eluted samples were carried out in flat-bottom microtitre plates by using BAPNA as specific substrate; the assay products were measured at $405 \mathrm{~nm}$, as previously described. ${ }^{2}$ Chymotrypsin inhibitory activity (CIA) evaluation of eluted samples was carried out by using BTEE as specific substrate, as previously described. ${ }^{17}$ Purified IBB1 and IBBD2 were extensively dialysed against distilled water at $4{ }^{\circ} \mathrm{C}$ overnight and freeze-dried.

\subsection{Measurement of protease inhibitory activities}

The major BBI isoinhibitors, IBB1 and IBBD2, were assessed for TIA and CIA. TIA was measured using a modified smallscale quantitative assay with BAPNA as specific substrate, and using $50 \mathrm{mM}$ Tris, pH 7.5 as enzyme assay buffer. ${ }^{18}$ CIA was measured using BTEE as specific substrate as described above.

\subsection{Glycation reaction of soybean BBI isoinhibitors}

For an efficient glycation, previous studies have fixed a molar ration carbohydrate to free amine groups in the protein around 6 considering both Lys and Arg residues and amino terminal groups. ${ }^{19}$ Taking into account the number of theoretical glycation sites in BBI the molar ratio glucose : BBI was set at 36 in our model system. The purified proteins, IBB1 and IBBD2, and glucose were dissolved in $0.1 \mathrm{M}$ phosphate buffer, $\mathrm{pH} 7$, at concentrations of 1 and $0.8 \mathrm{mg} \mathrm{mL}^{-1}$, respectively. The samples of the mixed solution were pipetted into capped pirex test tubes, and heated in a water bath at $95{ }^{\circ} \mathrm{C} .100 \mu \mathrm{L}$ of the mixture was sampled at different time points 30, 60, 90, and 120 minutes. In addition, control experiments were performed with the protein samples stored at $95{ }^{\circ} \mathrm{C}$ without glucose during the same period. The protein preparations were dialysed extensively against distilled water at $4{ }^{\circ} \mathrm{C}$ and kept at $-20{ }^{\circ} \mathrm{C}$ until use. At least six independent glycation experiments were carried out; inhibitory activity was measured in triplicates for each time point. To study how the treatment affected the inhibitory activities of BBI proteins, the percentage of trypsin and chymotrypsin inhibitory activity during heat treatment in the presence or absence of glucose was considered, being $100 \%$ of inhibitory activity that of the purified protein without heating. The data were analysed statistically by the Bonferroni's test to compare means and statistical significance was set at $p<0.05$. 


\subsection{Mass peptide fingerprinting of glycated soybean BBI isoinhibitors}

Soybean protease inhibitors were dissolved in NuPAGE lithium dodecyl sulphate sample buffer (Invitrogen, Paisley, UK) and separated by electrophoresis on Novex $4-12 \%$ Bis-Tris pre-cast gels using 2- $\mathrm{N}$-morpholine-ethane sulphonic acid (Nu-PAGE MES, Invitrogen) as running buffer. Immediately before use, samples were reduced with dithiothreitol (DTT) and NuPAGE antioxidant added to the upper buffer chamber to prevent reoxidation of reduced proteins during electrophoresis. Bands were excised from Colloidal Blue (Invitrogen)-stained gels and subjected to in-gel trypsin digestion. Peptide fragments from digested proteins were desalted and concentrated using C-18 ZipTip columns (Millipore, Madrid, Spain) and then, loaded directly onto the matrix-assisted laser desorption/ionisation (MALDI) plate, using $\alpha$-cyano-4-hydroxycinnamic acid as the matrix for MALDI-mass spectrometry (MS) analysis. MS spectra were obtained automatically in a 4700 Proteomics Analyzer (Applied Biosystems, Cheshire, UK) operating in reflectron mode with delayed extraction. Peptide mass data were used for protein identification against the MS protein sequence database (http://www.matrixscience.com). Using the software FindPept (http://web.expasy.org/findpept/), in silico analysis was performed on the masses obtained by trypsin digest of IBB1 and IBBD2 of glycated or non-glycated samples. FindPept can identify unmatched masses resulting from unspecific cleavages in peptide fingerprint protein identifications.

\subsection{Cell viability assays}

Human colorectal adenocarcinoma HT29 cells were maintained by serial passage in $75 \mathrm{~cm}^{2}$ plastic culture flasks. HT29 cells were cultured in DMEM, supplemented with fetal bovine serum (10\%), $2 \mathrm{mM}$ glutamine and 1\% antibiotic-antimicotic solution (Sigma, A5955), all at final concentration. Ninety-sixwell microtitre plates were inoculated at a density of 2000 HT29 cells per well in $200 \mu \mathrm{l}$ of growth media. Plates were incubated under $5 \% \mathrm{CO}_{2}$ in humidified air for $24 \mathrm{~h}$ to allow the cells to adhere to the wells. Glycated and non-glycated IBB1 and IBBD2 proteins were dissolved in growth media at 125,250 and $500 \mu \mathrm{g} \mathrm{mL}{ }^{-1}$ and added to the cells under sterile conditions. Control cells received neither form of BBI. After the incubation period of $96 \mathrm{~h}$, cell viability was evaluated using NR (neutral red, 3-amino-7-dimethylamino-2-methylphenazine hydrochloride) cytotoxicity assay, based on the ability of viable uninjured cells to incorporate and actively bind NR, a supravital dye, into lysosomes. Cells were stained in NR solution $\left(2 \mathrm{~h}\right.$ at $\left.37{ }^{\circ} \mathrm{C}\right)$, followed by cell fixation $(0.5 \%$ formaldehyde, $0.1 \% \mathrm{CaCl}_{2}$ for $30 \mathrm{~s}$ ) at room temperature. Plates were washed by two brief immersions in PBS (0.01 M-sodium phosphate buffer, $0.15 \mathrm{M} \mathrm{NaCl}$ ) and the dye extracted from the viable cells using an acidified ethanol solution (50\% ethanol, $1 \%$ acetic acid) overnight at $4{ }^{\circ} \mathrm{C}$. The absorbance of the solubilised dye was quantified at OD550 nm using a Bio-Rad Model 550 microplate reader (Bio-Rad). Cell viability data, expressed as a percentage of the values deter- mined for control cells grown in the absence of either form of BBI, were obtained from at least three independent experiments ( $n \geq 4$ per experiment). The data were analysed statistically by the Bonferroni's test to compare means and statistical significance was set at $p<0.05$.

\section{Results and discussion}

\subsection{Purification and characterization of Bowman Birk isoinhibitors from soybean}

Commercially available BBI from soybean is a mixture of isoforms with different inhibitory activities due to amino acid sequence variation within their inhibitory domains. ${ }^{1}$ The main BBI isoforms object of this study, IBB1 and IBBD2, were fractionated by Monos cation exchange chromatography; the elution pattern of the mixture of protease inhibitors was monitored by TIA and CIA measurements (Fig. 1A). Two major chromatographic peaks were resolved; peak I was collected in the unbound fraction, while peak II was eluted with a gradient of $\mathrm{NaCl}$ in the range 0.04-0.08 M. Peak I exhibited TIA and CIA activity whereas peak II showed TIA activity only. The chromatographic fractions were pooled individually, dialyzed extensively against water and freeze-dried. Both the commercial mixture and purified BBI proteins were analysed by SDS-PAGE (Fig. 1B). Proteins from peaks I and II showed apparent molecular mass in the range $8-10 \mathrm{kDa}$. The identity of the proteins was confirmed by mass peptide fingerprinting (Fig. 1C). Peak I was identified as Bowman-Birk proteinase inhibitor type I (IBB1, SwissProt accession number: P01055) and Peak II as Bowman-Birk proteinase inhibitor-DII (IBBD2, SwissProt accession number: P01064). Identification of the proteins was unequivocal since IBB1 sequence coverage was $92.9 \%$ and IBBD2 was $85.5 \%$. Amino acid sequences of both proteins was compared (Fig. 1D). Both proteins contain 14 conserved cysteine residues and two inhibitory domains. Both inhibitory domains of IBBD2 are very conserved, with arginine (R) at the $\mathrm{P} 1$ position, following the nomenclature of Schechter \& Berger (1967), ${ }^{20}$ that determines inhibitory activity for trypsin; both inhibitory domains only differ in positions $\mathrm{P} 2{ }^{\prime}$ and $\mathrm{P} 4{ }^{\prime}$. IBB1 has two inhibitory domains with wider variation; position $\mathrm{P} 1$ at the N-terminal domain is occupied by a lysine (K) which determines inhibitory activity for trypsin whereas in the C-terminal domain such position is occupied by leucine (L), which determines chymotrypsin inhibitory activity. ${ }^{1}$ Since protein glycation takes place when reducing sugars become attached to amino groups of proteins it was important to define the potential glycation spots in both sequences. All free amino groups can give rise to glycation products, but lysine (K) and arginine (R) side chains preferentially participate in the reaction. The most common reducing sugar in vivo is glucose, which reacts with $\mathrm{N}$-terminal amino acids or with free amino groups on lysine and arginine residues. There are potentially 7 sites that can be glycated in IBB1 and 9 sites in IBBD2 (Fig. 1D). Both isoinhibitors share four potential glycation sites K6/14, R28/36, K37/45 and K63/71 (IBB1/IBBD2 posi- 
A

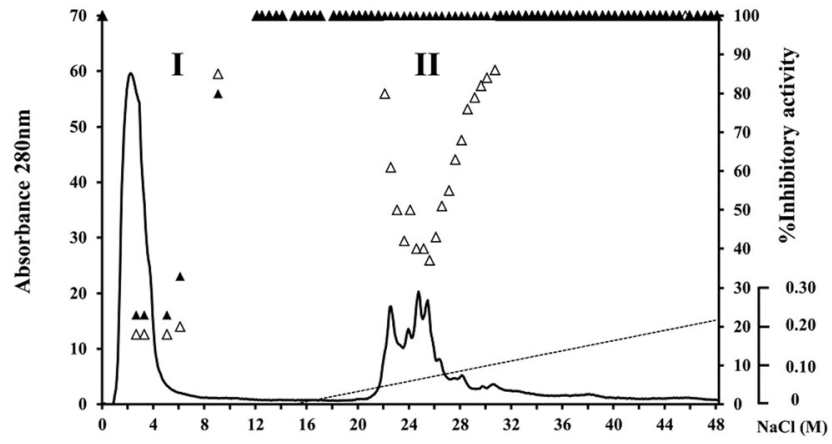

C

\begin{tabular}{cl}
\hline Peak ID & Protein name \\
\hline I & Bowman-Birk proteinase inhib \\
& $\begin{array}{l}\text { Bowman-Birk type proteinase } \\
\text { inhibitor D-II }\end{array}$
\end{tabular}

D

Swiss-Prot
$\begin{aligned} & \text { Accesion } \\ & \text { number }\end{aligned}$

P01055

IBB1_SOYBEAN
P01064
B

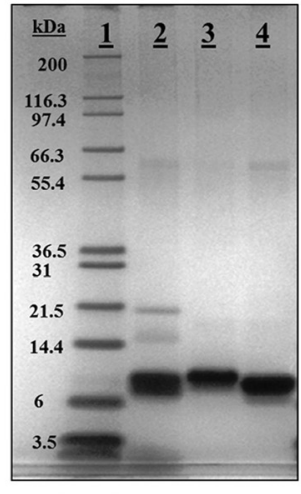

Sequence

coverage

(\%) peptides Score

IBBD2_SOYBEAN

$85.5 \%$

9

107

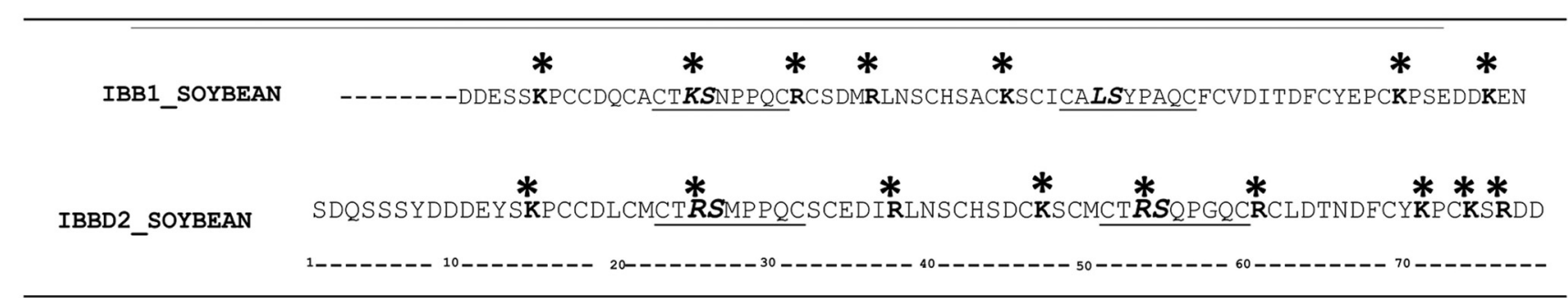

Fig. 1 (A) Elution profile of BBI isoinhibitors from soybean on a MonoS 5/50 GL cation exchange column. Absorbance (mAU) at 280nm of the chromatographic elution and the linear gradient of $\mathrm{NaCl}(0-0.22 \mathrm{M}$ ) are shown (solid and dotted lines, respectively). Using $N$ - $\alpha$-benzoyl-DL-arginine- $p$ nitroanilide (BAPNA) and $N$-benzoyl-L-tyrosine ethyl ester (BTEE) as specific substrates, the trypsin $(\triangle)$ and chymotrypsin $(\mathbf{\Delta})$ inhibitory activities, measured on every fraction are shown. (B) SDS-PAGE under denaturing and reducing conditions of BBI commercial mixture (lane 2) and peaks I and II (lanes 3 and 4, respectively), following the chromatography step. Molecular weight (MW) markers are shown in lane 1. (C) Protein identification deduced by peptide mass fingerprinting (Swiss-Prot database, Swiss Institute of Bioinformatics). (D) Amino acid sequences of proteins with inhibitory domains underlined. P1-P1'are the reactive peptide bond sites, in bold text. Lys (K) and Arg (R) at position P1 determine specificity for trypsin and Leu (L) for chymotrypsin. Possible glycation sites are indicated using asterisks.

tions). The fifth potential site shared is at position P1 within the N-terminal inhibitory domain, K16 for IBB1 an R24 for IBBD2. There are two additional sites in IBB1: R23 and K69; and four sites in IBBD2: R58, K68, R73 and R51, this last one is at position $\mathrm{P} 1$ of the C-terminal inhibitory domain. Although these are the potential glycation sites we need to bear in mind that their location in the three-dimensional protein structure will determine their glycation and indeed this would not be homogenous. ${ }^{21}$

\subsection{Protease inhibitory activity of IBB1 and IBBD2 after heating in the presence/absence of glucose}

To study the effect of glycation in protease inhibitory activities of IBB1 and IBBD2, glycation reactions were prepared in a concentration ratio $1: 0.8(\mathrm{w}: \mathrm{w}$; protein: glucose). The mixtures were heated at $95{ }^{\circ} \mathrm{C}$ for $120 \mathrm{~min}$, taking aliquots every $30 \mathrm{~min}$ to monitor the reaction. In order to evaluate the effect of temperature only, identical treatment was applied to the protease inhibitors in the absence of glucose. The protease inhibitory activity at time 0 was considered $100 \%$ of the total activity and percentage of inhibitory activity was calculated at different time points every 30 minutes. Native IBBD2 showed TIA of $4819 \pm 110$ units per milligram of protein and no CIA activity whereas IBB1 showed TIA of $3828 \pm 101$ and CIA of $2917 \pm 292$ units per milligram of protein. Evolution of trypsin and chymotrypsin inhibitory activities was measured and the profile of inactivation of the two isoinhibitors was considerably different from each other. None of the thermal treatments, in the presence or absence of glucose, caused an important variation in the protease inhibitory activities of IBB1 over time (Fig. 2A).

After $120 \mathrm{~min}$ at $95{ }^{\circ} \mathrm{C}$, only $25 \%$ of trypsin inhibitory activity was lost in IBB1 in the absence of glucose. These 
A
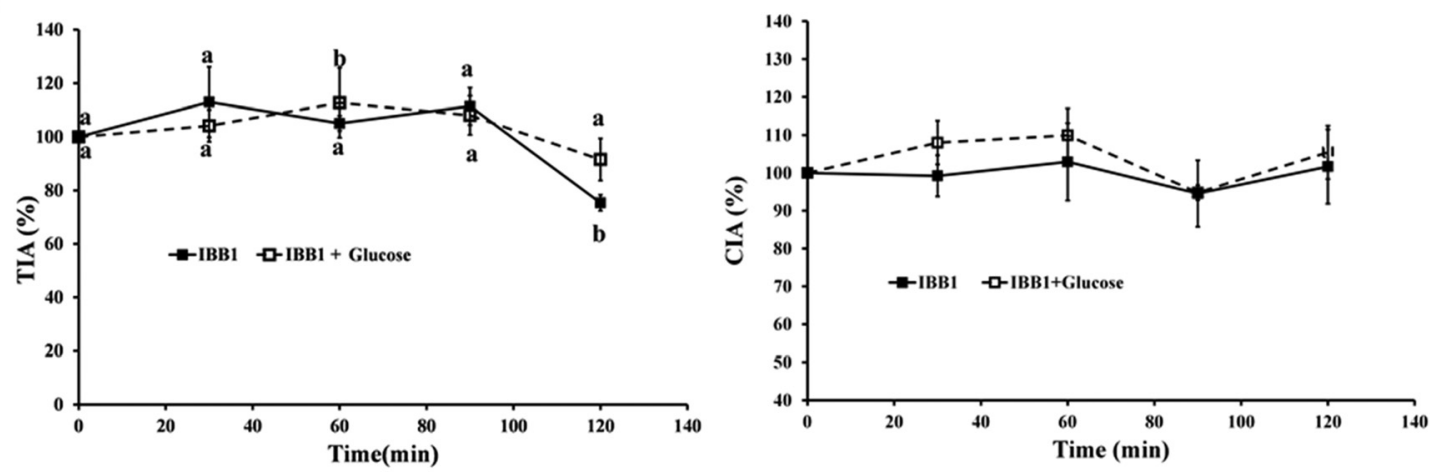

B

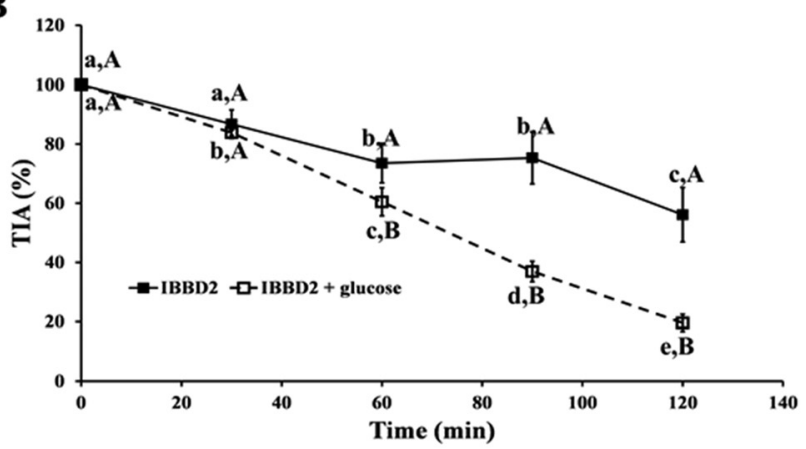

Fig. 2 Inhibitory activity of BBI isoinhibitors. (A) Percentage of trypsin and chymotrypsin inhibitory activity of IBB1 during heat treatment in the presence or absence of glucose, being $100 \%$ of inhibitory activity that of the purified protein without heating. (B) Percentage of trypsin inhibitory activity of IBBD2 during heat treatment in the presence or absence of glucose, being $100 \%$ of inhibitory activity that of the purified protein without heating. Data are the means of at least six independent experiments, each having three technical triplicates; bars represent standard deviations. Different letters denote significant differences between samples $(p<0.05)$; Bonferroni's test. Lower case letters are used to compare activity over time; uppercase letters are used to compare activity between glycated and not glycated inhibitors at same time point.

results confirm the thermal stability of IBB1 from soybean as previously described by several researchers. ${ }^{22-24}$ In another study, IBB1 was reported to retain $75 \%$ of its activity after $360 \mathrm{~min}$ at $100{ }^{\circ} \mathrm{C}$ while soy extract lost its ability to inhibit trypsin/chymotrypsin faster under similar conditions, assuming that the interaction of the inhibitors with other components in the extract could be responsible for this loss of activity. ${ }^{23}$ Similar results were recently reported where BBI concentrate was inactivated faster than pure IBB1; this was attributed, at least partially, to the protein aggregation of the concentrate that occurred during heating. ${ }^{15}$ BBIs from other legumes have also been reported to be extraordinarily stable when subjected to boiling, under either neutral or acidic conditions. ${ }^{25}$ Circular dichroism spectroscopy and fluorescence studies have revealed that the conserved cysteine residues, involved in the seven intramolecular disulphide bridges present in the protein, provide extreme stability to high temperatures and resistance to proteolysis and help to maintain the structural and functional features of the inhibitory domains in BBI from different species. ${ }^{24}$ Interestingly, the presence of glucose did not influence either trypsin or chymotrypsin inhibitory activity of IBB1. The chymotrypsin inhibitory domain seems even more stable since its inhibitory activity is not affected at all by temperature after treatment for $120 \mathrm{~min}$ at $95{ }^{\circ} \mathrm{C}$ (Fig. 2A). The crystal structure of IBB1 reveals that the protein has five disulphide-bonds exposed to the exterior and two other buried; IBB1 also shows two exposed hydrophobic patches making a very stable dimeric conformation. ${ }^{26}$ The extremely stable structure of IBB1 with hydrogen bonds between lysine resides and other amino acids may block the contact of the glucose with the target amino acid obstructing its glycation. ${ }^{27}$ The thermal resistance pattern of IBBD2 obtained for trypsin inhibitory activity was very different to those obtained for IBB1; a great loss of inhibitory activity was observed over time, being this loss significantly higher in the presence of glucose. After $90 \mathrm{~min}$ of treatment, in the presence of glucose, the trypsin inhibitory activity observed for IBBD 2 was $37 \%$ with respect to control and after $120 \mathrm{~min}$ it was less than $20 \%$ compared to control (Fig. 2B). Even though the sequences of IBB1 and IBBD2 are very similar, there must be structural features responsible for the loss of activity of IBBD2. Glycation can cause loss of the inhibitory activity of the protein by altering its structure. ${ }^{27}$ The proteolytic action of trypsin at the C-terminal lysine and arginine residues within the peptide chain is hindered when the side chain of these residues have been modified as a consequence of the thermal food processing. ${ }^{28}$ In a similar way, the trypsin linkage in the two inhibitory domains of IBBD2, containing an arginine residue, might 
be partially blocked due to the glycation induced by the heat treatment in the presence of glucose. Since these inhibitory domains determine inhibitory activity of trypsin in IBBD2, such inaccessibility results in a decrease in TIA activity. Kato and Matsuda (1997) studied the inactivation profiles by glycation of four different protease inhibitors and observed that it depends on the type of amino acid present within the inhibitory domain. ${ }^{12}$ The trypsin inhibitory activities of lysine-type inhibitors decreased gradually during the incubation periods. The loss of free amino groups in BBI proteins resulted in the complete inactivation of its trypsin inhibitory activity. These authors suggested that not all lysine are equally susceptible to glycation. The glycation process used by Kato and Matsuda (1997) was extremely long in time (15 days) and makes it difficult to compare with our results but it points out the fact that the amino acids adjacent to the inhibitory domains might play a key role in the stability of the inhibitory activity of the BBI. Our previous studies suggest that glycation under thermal treatment might be responsible for decreasing protease inhibitory activities in soymilks; ${ }^{14}$ similar effects were observed when soybean BBI was added to orange juice prior thermal sterilization. ${ }^{13}$ Although glycation of the reactive sites within the inhibitory domains could be involved, neither the isoinhibitor nor the glycation pattern of their amino acid sequences was reported.

\subsection{Analysis of BBI glycation products}

To confirm that glycation of the isoinhibitors was taking place, mass spectra was acquired by MALDI-TOF-MS analysis of the samples, in the presence or absence of glucose, after 90 min of heating (Fig. 3). A molecular ion of $7860 \mathrm{~m} / \mathrm{z}$ corresponding to the MW expected on the basis of the known soybean IBB1 sequence (UniprotKB/SwissProt accession number P01055 on http://www.expasy.ch protein data base) was found after heating the protein in the absence of glucose (Fig. 3A). With identical treatment, IBBD2 spectra revealed a molecular ion of similar size $7859.696 \mathrm{~m} / \mathrm{z}$, smaller than that expected one from its theoretical sequence (that is, $9468 \mathrm{Da}$ ) (UniprotKB/SwissProt accession number P01064) but closed to the obtained sequence (data not shown). This might be the result of the well-reported post-translational processing at the $\mathrm{N}$ - and $\mathrm{C}$-terminal ends of the protein.

Glycation mainly occurs on the primary amines of the $\mathrm{N}$-terminal residues and the $\varepsilon$ amine of lysine and arginine side chains. The progress of the glycation reaction can be detected using MS as an increase in mass over that of the protein under investigation. The change in mass will correspond to the addition of a glucose moiety with the concomitant loss of a water molecule $\left(\Delta M_{\mathrm{r}}=162\right)$. When IBB1 and IBBD2 were heated for $90 \mathrm{~min}$ in the presence of glucose, MALDI mass spectra showed a great heterogeneity of ions because different glycated forms of the proteins coexisted in the sample solution; thus, reported ion masses corresponded to mass increase of one, two, three or four added glucoses (Fig. 3B).

During the glycation process, and in order to explain the reported protease inhibitory activities where IBB1 was mostly unaffected by glycation while IBBD2 was significantly affected, we attempted to identify the glycated residues in both proteins. For this, untreated and treated proteins with glucose were analysed by MALDI TOF/TOF and the spectra obtained
A

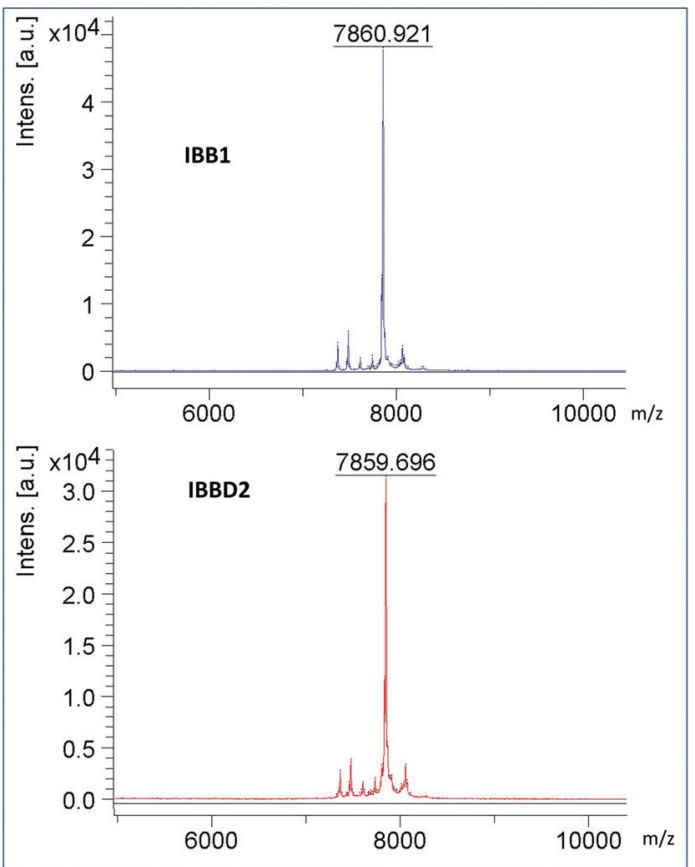

B

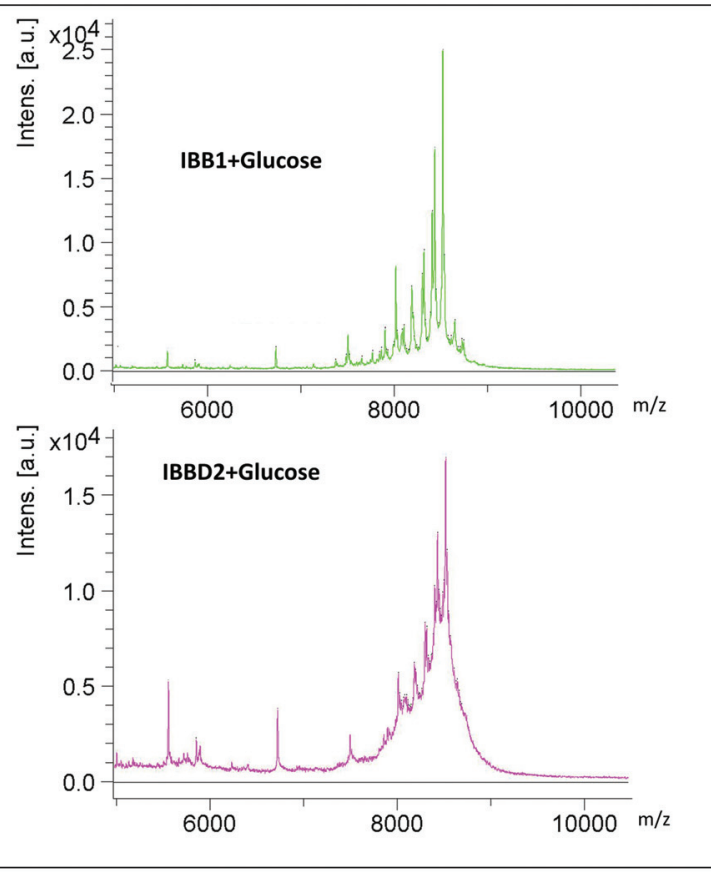

Fig. 3 MALDI-TOF mass spectra of purified IBB1 and IBBD2 from soy. (A) Protein spectra after heating at $95^{\circ} \mathrm{C}$ for 90 minutes; (B) protein spectra of glycated proteins after heating at $95^{\circ} \mathrm{C}$ for 90 minutes in the presence of glucose. 
was manually compared between samples. The amino acid sequences of the fragments present only in glycated samples were studied by FindPept, a software tool that identifies masses resulting from unspecific proteolytic cleavage as usually happens in glycated samples. Some of the peptides analysed had more than one potential glycation site but the majority had only one glycated residue. In the case of IBB1, six of the fractionated peptides, exclusively present in the glycated samples, could have a glycated lysine at P1 position (K16) (Table 1). Among them, only the fragment with $1856 \mathrm{Da}$ has as a unique option of glycation in this essential amino acid for the inhibitory activity of the protein. The other analysed fragments whose $[\mathrm{M}+\mathrm{H}]$ values were 2418, 2547, 2606, 2670 and $2709 \mathrm{Da}$, have additional potential glycation sites and only one implies the K16. The other sequences correspond to glycation possibilities out of the inhibitory domain (R23 and R28). For the rest of fragments analysed, the deduced sequences correspond to amino acids always out of the inhibitory domains. These results could explain why IBB1 does not lose the inhibi-

Table 1 Glycated peptides of IBB1 assigned by MALDI-MS analysis. Glycated amino acids are in bold. Amino acids in the inhibitory domains are in cursive and residues that react with proteases are underlined

\begin{tabular}{|c|c|}
\hline $\begin{array}{l}\text { Experimental } \\
\text { mass }[\mathrm{M}+\mathrm{H}]^{a}\end{array}$ & Putative peptide sequence \\
\hline 1215 & (P)PQCRCSDM(R) \\
\hline 1304 & (R)/CSDMRLNSC $(\mathrm{H})$ \\
\hline 1360 & $\begin{array}{l}\text { (S)KPCCDQCAC(T) } \\
\text { (C)KSCICALSYP(A) }\end{array}$ \\
\hline 1547 & (D)DESSKPCCDQC(A) \\
\hline 1830 & $\begin{array}{l}\text { (C)SDMRLNSCHSACKS(C) } \\
\text { (C)SDMRLNSCHSACKS(C) }\end{array}$ \\
\hline 1856 & (K)PCCDQCACT $\underline{K S} N P(Q)$ \\
\hline 2147 & (M)RLNSCHSACKSCICA $(L)$ \\
\hline 2161 & (K)/SNPPQCRCSDMRLNS(C) \\
\hline 2353 & (C)KSCICALSYPAQCFCVDI(T) \\
\hline 2418 & $\begin{array}{l}\text { (D)QCACT } \overline{K S} N P P Q C R C S D M R /(\mathrm{L}) \\
\text { (D)QCACTKS } N P P Q C R C S D M R /(\mathrm{L}) \\
\text { (D)QCACTKS } N P P Q C R C S D M R /(\mathrm{L})\end{array}$ \\
\hline 2547 & $\begin{array}{l}\text { (D)QCACT } \overline{K S} N P P Q C R C S D M R L(N) \\
\text { (D)QCACTKS NPPQCRCSDMRL(N) } \\
\text { (D)QCACT } \underline{K S} N P P Q C R C S D M R L(\mathrm{~N})\end{array}$ \\
\hline 2570 & $\begin{array}{l}\text { (R)/CSDMRLNSCHSACKSCIC(A) } \\
\text { (C)KSCICALSYPAQCFCVDITD(F) }\end{array}$ \\
\hline 2595 & $\begin{array}{l}\text { (P)QCRCSDMRLNSCHSACKSC(I) } \\
\text { (P)QCRCSDMRLNSCHSACKSC(I) } \\
\text { (P)QCRCSDMRLNSCHSACKSC(I) } \\
\text { (S)DMRLNSCHSACKSCICALSY }(P) \\
\text { (S)DMRLNSCHSACKSCICALSY(P) }\end{array}$ \\
\hline 2606 & $\begin{array}{l}\text { (C)ACTKSNPPQCRCSDMRLNS(C) } \\
\text { (C)ACTKS NPPQCRCSDMRLNS(C) } \\
\text { (C)ACTKSNPPQCRCSDMRLNS(C) }\end{array}$ \\
\hline 2670 & $\begin{array}{l}\text { (A) } C T \overline{K S N} P P Q C R C S D M R L N S C H(S) \\
\text { (A) } C T \overline{K S} N P P Q C R C S D M R L N S C H(S) \\
\text { (A) } C T \underline{K S} N P P Q C R C S D M R L N S C H(S)\end{array}$ \\
\hline 2696 & (C)HSACKSCICALSYPAQCFCV D(I) \\
\hline 2709 & 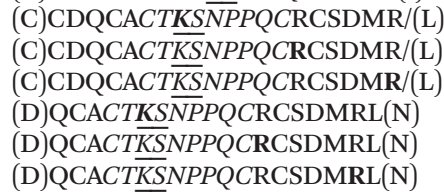 \\
\hline
\end{tabular}

${ }^{a}$ Monoisotopic mass value.

$24-41$

$37-56$

$21-39$

$26-45$

$13-31$

$14-33$

$33-53$ tory activity for trypsin during the glycation process since K16 seems less prone to be glycated. The C-terminal inhibitory domain has a leucine (L) at P1 position determining chymotrypsin inhibition and would not be affected by the glycation process. For IBBD2, a higher number of glycated fragmented peptides was obtained (Table 2). Both inhibitory domains have potential glycated sites at P1 position (R24 and R51). The number of deduced sequences with $\mathrm{P} 1$ glycated is higher than in IBB1. Peptides with masses 1574.55, 1865.74 and 2776 Da have the arginine at P1 (R24) within the N-terminal inhibitory domain likely glycated. In all the possible sequences for fragmented peptides 1930.8, 2292.87, 2326.87, 2611.963, 2644.92 and $2831 \mathrm{Da}$, there is always one with the arginine at P1 (R51)

Table 2 Glycated peptides of IBBD2 assigned by MALDI-MS analysis. Glycated amino acids are in bold. Amino acids in the inhibitory domains are in cursive and residues that react with proteases are underlined

Experimental $\operatorname{mass}[\mathrm{M}+\mathrm{H}]^{a}$ Putative peptide sequence Position 1574.55 1808.74 1865.74

(D)LCMCTRSMPPQ $(C)$

(L)NSCHSDCKSCMCT(R)

(K)PCCDLCMCTRSMP(P)

(P)CCDLCMCTR $\overline{S M} P P(Q)$

(C)MCTRSQPG $\overline{Q C R C L D(T)}$

1930.8

(C)MCTRS $Q P G Q C R C L D(T)$

2102.87

2251.89

(Q)SSSYDDDEYSKPCCDL(C)

(D)IRLNSCHSDCKSCMC(T)

(S)YDDDEYSKPCCDLCMC $(T)$

(L)NSCHSDCKSCMCTRSQP(G)

(L)NSCHSDCKSCMCTRS $Q P(G)$

(E)YSKPCCDLCMCTR $\overline{S M}(P)$

(R)/LNSCHSDCKSCMCTRSQ $(P)$

(R)/LNSCHSDCKSCMCTRS $Q(P)$

2326.87

(R)/LNSCHSDCKSCMCTRS $(Q)$

(G)QCRCLDTNDFCYKPCK/(S)

(G)QCRCLDTNDFCYKPCK/(S)

(G)QCRCLDTNDFCYKPCK/(S)

2386

(C)CDLCMCTRSMPPQCSCE(D)

(S)QPGQCRCLDTNDFCYK(P)

2611.963

(Q)CSCEDIRLNSCHSDCKSC(M)

(Y)SKPCCDLCMCTRSMPPQC(S)

(S)KPCCDLCMCTR $\overline{S M} P P Q C S(\mathrm{C})$

(M)PPQCSCEDIRLNSCHSDCKS(C)

(M)PPQCSCEDIRLNSCHSDCKS(C)

(D)IRLNSCHSDCKSCMCTRS $(Q)$

(D)IRLNSCHSDCKSCMCTRS $(Q)$

(D)IRLNSCHSDCKSCMCT RS $(Q)$

2642.91

(K)PCCDLCMCTRSMPPQCSCE(D)

(S)QPGQCRCLDTNDFCYKPC(K)

(Q)PGQCRCLDTNDFCYKPCK/(S)

(Q)PGQCRCLDTNDFCYKPCK/(S)

(Q)PGQCRCLDTNDFCYKPCK/(S)

2644.92

(P)CCDLCMCTRSMPPQCSCED(I)

(E)YSKPCCDLCM $C T R S M P P Q C(\mathrm{~S})$

(E)YSKPCCDLCMCTRSMPPQC(S)

(I)RLNSCHSDCKSCMCTRS $(Q)$

2776

(D)EYSKPCCDLCMCTRSMPPQ(C)

(Y)DDDEYSKPCCDLC $\bar{M} C T R S M P P(Q)$

(Y)DDDEYSKPCCDLCMCTRSMPP(Q)

(K)/SCMCTRSQPGQCRCLDTNDFC(Y)

(K)/SCMCT $\overline{R S} Q P G Q C R C L D T N D F C(Y)$

(E)DIRLNSCHSDCKSCMCTRSQPG(Q)

(E)DIRLNSCHSDCKSCMCTRSQPG(Q)

(E)DIRLNSCHSDCKSCMCT $\underline{R S} Q P G(Q)$

$19-29$

38-50

$15-27$

16-28

48-61

4-19

35-49

$7-22$

$38-54$

$12-26$

$37-53$

$37-52$

$56-71$

17-33

53-68

$30-47$

13-30

14-31

27-46

$35-52$

15-33

$53-70$

$54-74$

16-34

12-30

36-52

9-29

$8-28$

46-66

$34-55$

\footnotetext{
${ }^{a}$ Monoisotopic mass value.
} 
glycated. The 14 fragmented peptides observed exclusively in IBBD2 treated with glucose could have the amino acid at P1 position glycated and that would probably hinder the inhibitory activity of the protein. We have observed that not all lysine residues undergo glycation. It has been suggested that the immediate chemical environment of an amino group might modulate the glycation reaction; thus, close amino acids might interact at intra- or extra-molecular level. For example, the presence of a histidine or another lysine close to the lysine in the primary structure or tertiary structure promotes the glycation on this one. ${ }^{29}$ Studies with peptide models demonstrated that neighboring groups are essential in catalyzing glycation at lysine residues; thus, the positioning of strongly basic residues near lysine promotes rapid glycation. ${ }^{30}$

\subsection{Effect of glycation on antiproliferative activity of soybean BBI isoinhibitors}

Soybean BBI and homologous proteins appear to be promising chemopreventive agents within the gastrointestinal tract. ${ }^{31,32}$ Our previous studies have proven the capacity of BBI from different sources like pea, lentil and soybean to inhibit the growth of human colon cancer cells HT29 in a dose-dependent manner. ${ }^{1,33,34}$ Chemically inactive BBI, obtained by reducing and further alkylation of sulphydryl groups, were unable to inhibit serine proteases and would lose the capacity to inhibit the cell proliferation of human colon cancer cells probing the connection between these two actions of the BBI. ${ }^{1}$ Since the inactivation of BBI was carried out by disturbing the native conformation of the protein, it was not clear if the loss of the antiproliferative activity was a consequence of loss of the protein structure or the loss of the inhibitory activity. The results obtained later with recombinant BBI from pea and inactive derivative mutants having similar configuration demonstrated that there was a direct association between the inhibitory activity of serine proteases and the antiproliferative activity against HT29 colon cancer cells. ${ }^{10}$ As shown above, the inhibitory activity of BBI is not equally affected in both soybean BBI isoforms. While IBB1 is very resistant to temperature and glycation, IBBD2 can lose up to $80 \%$ of its trypsin inhibitory activity due to glycation and $40 \%$ due to the temperature (Fig. 2B). For all stated above, this loss of activity by glycation could have a negative effect over the antiproliferative activity of IBBD2 but not IBB1. In order to prove this hypothesis, human colon adenocarcinoma HT29 cells were cultured in the presence of glycated or not glycated BBI proteins at different concentrations $\left(0.125-0.500 \mathrm{mg} \mathrm{ml}^{-1}\right)$. Cell viability was monitored by the cytotoxic NR cell assay after 96 hours, established as the necessary time to observe a significant reduction in cell viability at all concentrations. ${ }^{1}$

A statistically significant $(P<0.05)$ and dose-dependent decrease on the growth of HT29 colon cells was observed after treatment with non-glycated and glycated IBB1 (Fig. 4A). The slight difference between cell growth in the presence of nonglycated and glycated IBB1 was not statistically different. The inhibition profile was very similar for glycated and non-glycated IBB1 showing that glycation of IBB1 did not have a
A

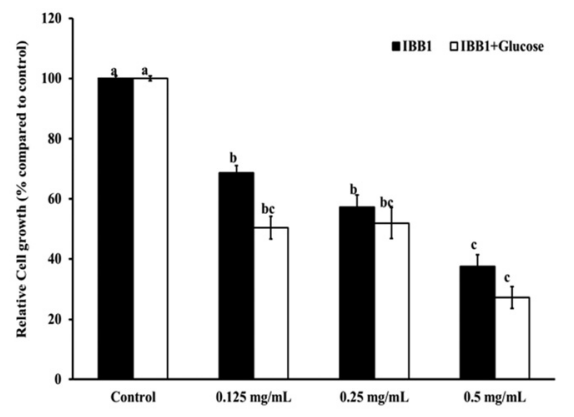

B

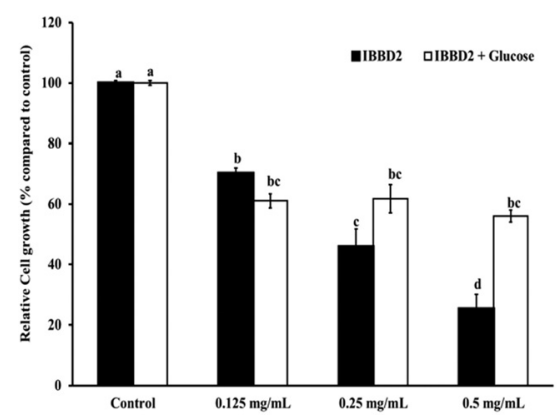

Fig. 4 Effects of BBI glycation on the in vitro growth of HT29 human colorectal adenocarcinoma cells. IBB1 and IBBD2 were previously heated for $90 \mathrm{~min}$ in the presence or absence of glucose. Controls have no inhibitor and cell viability was considered $100 \%$. (A) Percentage of cell growth treated with IBB1 (closed bars) and glycated IBB1 (open bars). (B) Percentage of cell growth treated with IBBD2 (closed bars) and glycated IBBD2 (open bars). Growth media were supplemented with protein in the concentration range $\left(0.125-0.500 \mathrm{mg} \mathrm{mL}^{-1}\right)$ and cells harvested after a period of $96 \mathrm{~h}$. Values are means of at least three independent experiments with five technical replicates, bars represent standard deviations. Mean values with different letters were significantly different $(P<0.05$; Bonferroni's test). Lower case letters are used to compare relative cell growth with different concentrations of inhibitor, uppercase letters are used to compare relative cell growth after treatment with glycated and not glycated inhibitors.

detectable impact over the anti-proliferative properties of the inhibitor. IBBD2 inhibited cell growth in a dose-dependent manner when it was not glycated, but the dose-response relationship was lost when this protein was glycated (Fig. 4B). Glycated IBBD2 exerted an inhibitory effect over HT29 cells growth even at lower concentrations, but this effect did not increase with higher IBBD2 concentrations.

These results evidence that glycation of IBBD2 has a negative effect over its anti-proliferative properties. These data clearly support that the anti-proliferative activity of IBBD2 on HT29 cells is mediated via protease inhibition; probably the inhibition of cell growth observed with glycated protein (around $40 \%$ of inhibition in all cases) is the result of the remaining trypsin inhibitory activity (37\%, Fig. 2B). Consequently, the maintaining of the colorectal chemopreventive properties of BBI present in soybean products, like soybean, after industrial thermal processing would relay over the lower susceptibility of IBB1 isoform to glycation, since the 
IBBD2 evidences higher trend to glycation reaction in the same controlled conditions. Moreover, unlike IBBD2, IBB1 glycation takes place in spots that are not critical for the protease inhibitor activity.

\section{Conclusions}

Our previous studies and others have hypothesized that glycation could block lysine within soybean BBI inhibitory domains and in turn might decrease protease inhibitory activity. ${ }^{14,35}$ Our molecular approach establishes in a clear and effective way that glycation reaction takes place in the two major BBI isoinhibitors isolated from soybean, IBB1 and IBBD2, affecting distinct glycation spots in each soybean BBI isoinhibitor and with differential implications for its functionality.

Our findings suggest that the manufacturing of soy-derived products by common procedures in the food industry involving heat treatment (e.g. UHT applied to soy beverages) would not induce complete loss of the chemopreventive function attributed to BBI in the large intestine, since IBB1 isoform preserves its activity after glycation. However, the IBBD2 isoform is very sensitive to the conditions of food manufacturing so that, in the presence of reducing sugars and with the application of thermal treatment, it would participate in glycation reactions affecting its inhibitory domains. This fact would give rise to a drop in TIA of IBBD2 isoform and thus to its intrinsic antiproliferative activity against colon cancer cells. A correct handling of the thermal processes applied to soy derivatives by the food industry would result in the preservation of the biological activity of the BBI and its beneficial role in the intestinal health.

\section{Conflicts of interest}

There are no conflicts to declare.

\section{Acknowledgements}

This work was financially supported by grants AGL2017-83772R (AEI/FEDER,UE) and 201640E139 funded by the Spanish Ministry of Science, Innovation and Universities, and the Spanish National Research Council (CSIC), respectively.

\section{Notes and references}

1 A. Clemente, F. J. Moreno, M. del C. Marín-Manzano, E. Jiménez and C. Domoney, The cytotoxic effect of Bowman-Birk isoinhibitors, IBB1 and IBBD2, from soybean (Glycine max) on HT29 human colorectal cancer cells is related to their intrinsic ability to inhibit serine proteases, Mol. Nutr. Food Res., 2010, 54, 396-405.

2 A. Clemente, E. Jimenez, M. C. Marin-Manzano and L. A. Rubio, Active Bowman-Birk inhibitors survive gastro- intestinal digestion at the terminal ileum of pigs fed chickpea-based diets, J. Sci. Food Agric., 2008, 88, 513-521.

3 P. Chen, J. Rose, R. Love, C. H. Wei and B. C. Wang, Reactive sites of an anticarcinogenic Bowman-Birk proteinase inhibitor are similar to other trypsin inhibitors, J. Biol. Chem., 1992, 267, 1990-1994.

4 P. Ramasarma, A. Rao and D. Rao, Role of Disulfide Linkages in Structure and Activity of Proteinase-Inhibitor from Horsegram (dolichos-Biflorus), Biochim. Biophys. Acta, Protein Struct. Mol. Enzymol., 1995, 1248, 35-42.

5 M. V. Trivedi, J. S. Laurence and T. J. Siahaan, The Role of Thiols and Disulfides on Protein Stability, Curr. Protein Pept. Sci., 2009, 10, 614-625.

6 B. A. Baxter, R. C. Oppel and E. P. Ryan, Navy Beans Impact the Stool Metabolome and Metabolic Pathways for Colon Health in Cancer Survivors, Nutrients, 2019, 11, 28.

7 A. Clemente and R. Olias, Beneficial effects of legumes in gut health, Curr. Opin. Food Sci., 2017, 14, 32-36.

8 A. Kennedy, P. Billings, P. Maki and P. Newberne, Effects of Various Preparations of Dietary Protease Inhibitors on Oral Carcinogenesis in Hamsters Induced by Dmba, Nutr. Cancer, 1993, 19, 191-200.

9 M. P. Utrilla, M. J. Peinado, R. Ruiz, A. Rodriguez-Nogales, F. Algieri, M. E. Rodriguez-Cabezas, A. Clemente, J. Galvez and L. A. Rubio, Pea (Pisum sativum L.) seed albumin extracts show anti-inflammatory effect in the DSS model of mouse colitis, Mol. Nutr. Food Res., 2015, 59, 807-819.

10 A. Clemente, M. C. Marín-Manzano, E. Jiménez, M. C. Arques and C. Domoney, The anti-proliferative effect of TI1B, a major Bowman-Birk isoinhibitor from pea (Pisum sativum L.), on HT29 colon cancer cells is mediated through protease inhibition, Br. J. Nutr., 2012, 108, S135-S144.

11 M. W. Poulsen, R. V. Hedegaard, J. M. Andersen, B. de Courten, S. Bugel, J. Nielsen, L. H. Skibsted and L. O. Dragsted, Advanced glycation endproducts in food and their effects on health, Food Chem. Toxicol., 2013, 60, 10-37.

12 Y. Kato and T. Matsuda, Glycation of proteinous inhibitors: Loss in trypsin inhibitory activity by the blocking of arginine and lysine residues at their reactive sites, J. Agric. Food Chem., 1997, 45, 3826-3831.

13 M. Amigo-Benavent, C. Nitride, L. Bravo, P. Ferranti and M. D. del Castillo, Stability and bioactivity of a BowmanBirk inhibitor in orange juice during processing and storage, Food Funct., 2013, 4, 1051.

14 M. C. Arques, S. Pastoriza, C. Delgado-Andrade, A. Clemente and J. A. Rufián-Henares, Relationship between Glycation and Polyphenol Content and the Bioactivity of Selected Commercial Soy Milks, J. Agric. Food Chem., 2016, 64, 1823-1830.

15 H. He, X. Li, X. Kong, Y. Hua and Y. Chen, Heat-induced inactivation mechanism of soybean Bowman-Birk inhibitors, Food Chem., 2017, 232, 712-720.

16 Z. Xu, Y. Chen, C. Zhang, X. Kong and Y. Hua, The HeatInduced Protein Aggregate Correlated with Trypsin Inhibitor Inactivation in Soymilk Processing, J. Agric. Food Chem., 2012, 60, 8012-8019. 
17 A. Clemente, D. A. MacKenzie, D. J. Jeenes and C. Domoney, The effect of variation within inhibitory domains on the activity of pea protease inhibitors from the Bowman-Birk class, Protein Expression Purif., 2004, 36, 106114.

18 C. Domoney and T. Welham, Trypsin inhibitors in Pisum: variation in amount and pattern of accumulation in developing seed, Seed Sci. Res., 1992, 2, 147-154.

19 F. J. Moreno, R. Lopez-Fandino and A. Olano, Characterization and functional properties of lactosyl caseinomacropeptide conjugates, J. Agric. Food Chem., 2002, 50, 5179-5184.

20 I. Schechter and A. Berger, On the size of the active site in proteases. I. Papain, Biochem. Biophys. Res. Commun., 1967, 27, 157-162.

21 J. O'Brien, P. A. Morrissey and J. M. Ames, Nutritional and toxicological aspects of the Maillard browning reaction in foods, Crit. Rev. Food Sci. Nutr., 1989, 28, 211-248.

22 M. Amigo-Benavent, A. Clemente, P. Ferranti, S. Caira and M. D. del Castillo, Digestibility and immunoreactivity of soybean $\beta$-conglycinin and its deglycosylated form, Food Chem., 2011, 129, 1598-1605.

23 C. M. DiPietro and I. E. Liener, Heat inactivation of the Kunitz and Bowman-Birk soybean protease inhibitors, J. Agric. Food Chem., 1989, 37, 39-44.

24 Y. V. Wu and D. J. Sessa, Conformation of Bowman-Birk Inhibitor, J. Agric. Food Chem., 1994, 42, 2136-2138.

25 M. A. Osman, P. M. Reid and C. W. Weber, Thermal inactivation of tepary bean (Phaseolus acutifolius), soybean and lima bean protease inhibitors: effect of acidic and basic pH, Food Chem., 2002, 78, 419-423.

26 R. H. Voss, U. Ermler, L. O. Essen, G. Wenzl, Y. M. Kim and P. Flecker, Crystal structure of the bifunctional soybean Bowman-Birk inhibitor at $0.28-\mathrm{nm}$ resolution - Structural peculiarities in a folded protein conformation, Eur. J. Biochem., 1996, 242, 122-131.
27 H. Huang, M. Zhao, Q. Ren and Y. Yang, Changes in inhibitory activity and secondary conformation of soybean trypsin inhibitors induced by tea polyphenol complexation, J. Sci. Food Agric., 2009, 89, 2435-2439.

$28 \mathrm{~J}$. M. Ames, Mass spectrometry to detect the site specificity of advanced glycation/lipoxidation end-product formation on protein: some challenges and solutions, Biochem. Soc. Trans., 2008, 36, 1051-1054.

29 D. J. Walton and B. H. Shilton, Site specificity of protein glycation, Amino Acids, 1991, 1, 199-203.

$30 \mathrm{~J}$. Venkatraman, K. Aggarwal and P. Balaram, Helical peptide models for protein glycation: proximity effects in catalysis of the Amadori rearrangement, Chem. Biol., 2001, 8, 611-625.

31 A. Clemente and M. C. Arques, Bowman-Birk inhibitors from legumes as colorectal chemopreventive agents, World J. Gastroenterol., 2014, 20, 10305.

32 A. Clemente, G. Sonnante and C. Domoney, Bowman-Birk Inhibitors from Legumes and Human Gastrointestinal Health: Current Status and Perspectives, Curr. Protein Pept. Sci., 2011, 12, 358-373.

33 P. Caccialupi, L. R. Ceci, R. A. Siciliano, D. Pignone, A. Clemente and G. Sonnante, Bowman-Birk inhibitors in lentil: Heterologous expression, functional characterisation and anti-proliferative properties in human colon cancer cells, Food Chem., 2010, 120, 1058-1066.

34 M. C. Marín-Manzano, R. Ruiz, E. Jiménez, L. A. Rubio and A. Clemente, Anti-carcinogenic soyabean Bowman-Birk inhibitors survive faecal fermentation in their active form and do not affect the microbiota composition in vitro, Br. J. Nutr., 2009, 101, 967-971.

35 N. Toro-Funes, J. Bosch-Fusté, M. L. Latorre-Moratalla, M. T. Veciana-Nogués and M. C. Vidal-Carou, Isoflavone profile and protein quality during storage of sterilised soymilk treated by ultra high pressure homogenisation, Food Chem., 2015, 167, 78-83. 